\title{
Support needed as 9 million children miss out on care
}

The British Dental Association has stressed government must meaningfully support practices to boost capacity, as new official figures underline the collapse in NHS access during the pandemic.

NHS dental statistics for England 2020/21 show the proportion of children seen by an NHS dentist in the last 12 months fell from $58.7 \%$ as of 31 March 2020 to $23 \%$ on 31 March 2021, meaning over 9 million missed out on care in the year following the first lockdown.

The BDA has emphasised that data are yet to capture the full impact of the pandemic access to services among adults, where attendance is measured every two years: $49.6 \%$ of adults were seen by an NHS Dentist in the 24 months up to April 2020, falling to $42.8 \%$ in the period up to April 2021.

Figures also show 12 million courses of treatment were delivered in 2020/21, a

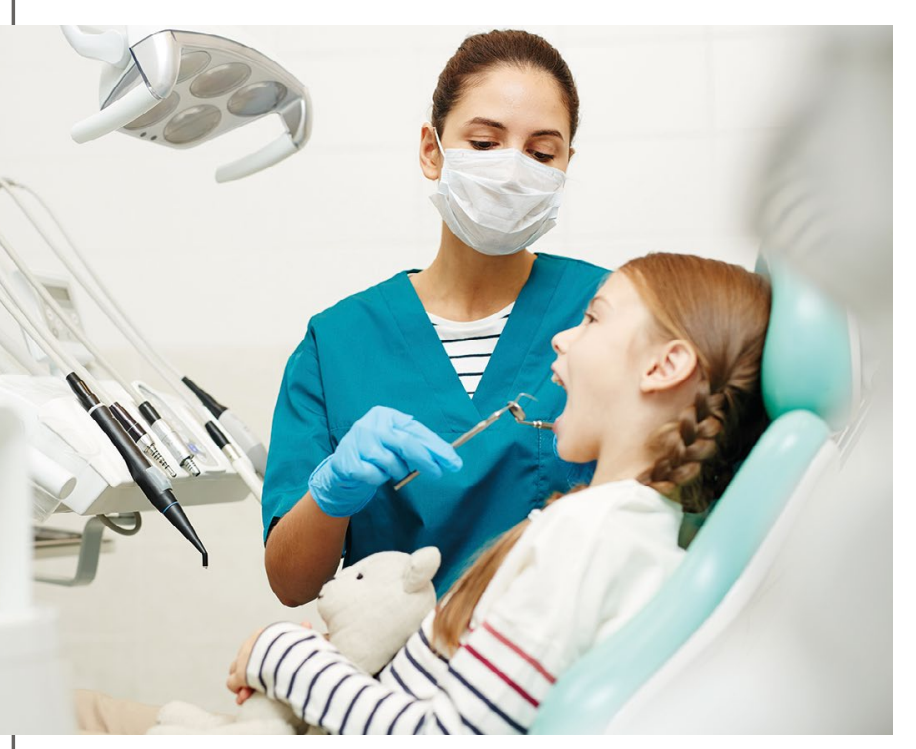

decrease of $69 \%$ compared to the previous year.

Over 30 million courses of NHS

treatment have now been lost since the first lockdown. Delays in diagnosis can mean poorer outcomes for patients, and can require more complex treatments.

Capacity across the service remains low. The BDA understands around half the NHS practices in England are not currently meeting controversial targets imposed by government that require them to hit $60 \%$ of pre-COVID-19 activity levels, and as a result will face financial penalties. Dentist leaders stress this reflects the limits of what's possible under strict infection control guidance, which remains largely unchanged since the resumption of routine care in June 2020. A 45\% target was imposed on 1 January 2021, days before the country entered a third national lockdown, rising to its current level on 1 April.

While every other UK nation has provided capital funding to help practices increase capacity through new high-volume ventilation systems, there has been no commitment from authorities in England. Investment here would quickly pay for itself through recovery of patient charge revenues.

The BDA is also seeking a clear roadmap to safely ease restrictions, including the instructions to maintain 'gaps' between appointments that have radically reduced patient volumes. While in response to calls all four UK Chief Dental Officers issued an unprecedented joint statement in June indicating a review would take place, there has been no meaningful relaxation of standard operating procedures.

Access problems were already widespread prior to the pandemic, with funds allocated to provide NHS care for barely half the population, and unmet need estimated at over 4 million people, or nearly 1 in 10 adults. With oral health inequality now expected to widen the BDA has urged the government to be ambitious and ensure recent pledges to reform NHS dentistry turn the page on both the current crisis and historic problems that have dogged the service.

Shawn Charlwood, Chair of the BDA's General Dental Practice Committee said: 'Millions are still missing out on dental care, and patients will be paying the price for years to come.

'Dentists in England have had capacity slashed by pandemic restrictions, and need help to get patients back through their doors. Sadly, while every other UK nation has committed funds, Westminster chose to impose targets that thousands of practices are now struggling to hit.

'To deliver for patients we need real support, and a clear roadmap to ease restrictions. But even before COVID-19 there simply wasn't enough NHS dentistry to go round. We cannot return to a 'business as usual' where access problems are the norm.'

\section{Date for the diary: Online event - Buying a dental practice}

A comprehensive guide to practice ownership When?

Buying a dental practice part 1 -

Preparing yourself on Wednesday 3

November 2021 (19:00 - 21:00)

Buying a dental practice part 2 - The legalities on Wednesday 10 November 2021 (19:00 - 21:00)

Buying a dental practice part 3 - Running a compliant practice on Wednesday 17
November 2021 (19:00 - 20:30)

Whether you are considering buying your own practice in the near or medium-term future, this three part online event will equip you with the tools to buy and run your own practice.

For further information or to book your place visit www.bda.org/events/ Pages/Buying-a-dental-practice-onlineNovember-2021.aspx. 